\title{
Very-small to large strain dynamic behaviour of unsaturated sand in a wide range of suction
}

\author{
Ali Akbar Karimezadeh ${ }^{1 *}$, Fardin Jafarzadeh ${ }^{2}$, Anthony Kwan Leung ${ }^{l}$ and Adel Ahmadinezhad ${ }^{2}$ \\ ${ }^{1}$ Dept. of Civil and Environmental Engineering, Hong Kong Univ. of Science and Technology, Kowloon, Hong Kong \\ ${ }^{2}$ Dept. of Civil and Environmental Engineering, Sharif Univ. of Technology, Tehran, Iran
}

\begin{abstract}
Shear modulus ( $G_{\max }$ at very small strain and $G$ at large strain) and constraint modulus at very small strain $(M)$ are important soil parameters for static and dynamic analysis in geotechnical applications. However, these dynamic properties of unsaturated soil are rarely reported. In this study, a cyclic simple shear apparatus was newly-modified for allowing both the shear and constrained moduli at both very small and large strains to be measured. Benders or ultrasonic sensors were embedded in an unsaturated soil sample for transmitting/receiving shear- and pressure-wave, respectively. Two very-small-strain tests were conducted to determine the $G_{\max }, M$ and soil damping ratio of a sand for a wide range of suction covering from the boundary-effect, transition and residual zone of the water retention curve of the sand. In addition, six large-strain cyclic simple shear tests were carried out to investigate $G$. The test results showed that $G_{\max }$ and $M$ were approximately constant before reaching the air-entry value, but there was a significant increase in $G_{\max }$ as the sand dried further. Yet, $M$ dropped within the transition zone, and interestingly when the suction was beyond the residual value, $M$ increased. $M$ along the wetting path was higher than that along the drying path. The damping ratio, on the other hand, first reduced before reaching the air-entry value, but it increased at the transition zone and then decreased within the residual zone. At large strain, $G / G_{\max }$ also increased as suction increased until reaching the residual zone, beyond which the normalised value show substantial decreased.
\end{abstract}

\section{Introduction}

Shear moduli at very small strain $\left(G_{\max }\right)$ and large strains $(G)$, are crucial parameters for predicting soil movements and response of soil-structure system when subjected to dynamic loading and when investigating the quality of earthworks via a spectral analysis of surface waves [1]. The constraint modulus $(M)$, on the other hand, was another important soil parameter when designing buried pipe lines and pavements [2, 3].

It is well-known that $G$ reduces with an increase in shear strain highly nonlinearly by orders of magnitude. Atkinson and Sallfors (1991) defined three ranges of strains in a soil: very small strains $(<0.001 \%)$, small strains (between $0.001 \%$ and $1 \%$ ), and large strains (> $1 \%$ ) [4]. Previous research has already shown that due to the compliance of a cyclic simple shear apparatus, only shear modulus and damping ratio at shear strain ranges from $0.1 \%$ to $1 \%$ can be reliably obtained [5]. However, soil behaviour at the very small strain range is difficult to be quantified and assessed. Measurement of $G_{\max }$ using piezoelectric transducers such as benders has been deemed successful, but as far as the authors are aware, the applications of this kind of transducers are largely limited in oedometers [6], torsional resonant column apparatus [7] and triaxial apparatus [8]. Cyclic simple shear apparatus, which is known to be effective in evaluating dynamic properties of soil, has rarely been equipped with piezoelectric transducers for measuring the very-small-strain response. Furthermore, while there has been extensive research in the literature in characterising the dynamic behaviour of saturated soil, investigation on unsaturated soil for a wide range of suction (i.e., from air-entry to residual suction and beyond) is limited. Hence, any effects of matric suction or degree of saturation $\left(S_{r}\right)$ on the stiffness properties (shear and constrained moduli), at both very small strain and large strains, are unclear.

The objective of this paper is to present some recent development of a cyclic simple shear apparatus for allowing the shear and constrained moduli at both very small and large strains to be measured. After elaborating the new features added to the apparatus, it was used to evaluate the effects of suction and $S_{r}$ on both shear and constrained moduli.

\section{Apparatus modification}

To study the very-small-strain to large-strain dynamic properties of unsaturated soils, a cyclic simple shear apparatus at the advanced geotechnical laboratory of Sharif University of Technology was newly-modified to measure the velocities of both $\mathrm{p}$ and s-wave (Fig. 1). The apparatus adopts the hanging-column and axis-

Corresponding author: aakarimzadeh@ connect.ust.hk 
translation techniques to control low and high ranges of matric suction of a soil specimen, respectively [9]. The apparatus is able to apply a controlled cyclic shear stress to a soil being subjected to a constant vertical loading. Dynamic soil behaviour at large strain range can thus be studied. The new modification made in this study was to allow the base pedestal to be mounted with both the high air-entry (1 bar) ceramic disc (for maintaining suction) and sensors (either bender or ultrasonic sensor for transmitting s- or p-wave, respectively (Fig. 2(a)). Another sensor (i.e., the receiver) was also mounted on the top pedestal (Fig. 2(b)) [10]. Based on the measurements of wave velocities at any given matric suction, $G_{\max }$ and $M$ can be determined based on the elastic theory of wave propagation in a homogenous porous material:

$$
\begin{gathered}
G_{\max }=\rho V_{s}^{2} \\
M=\rho V_{p}^{2}
\end{gathered}
$$

where $\rho$ is the soil bulk density, $V_{s}$ is the shear-wave (or s-wave) velocity; and $V_{p}$ is the constrained compression wave (or p-wave) velocity.

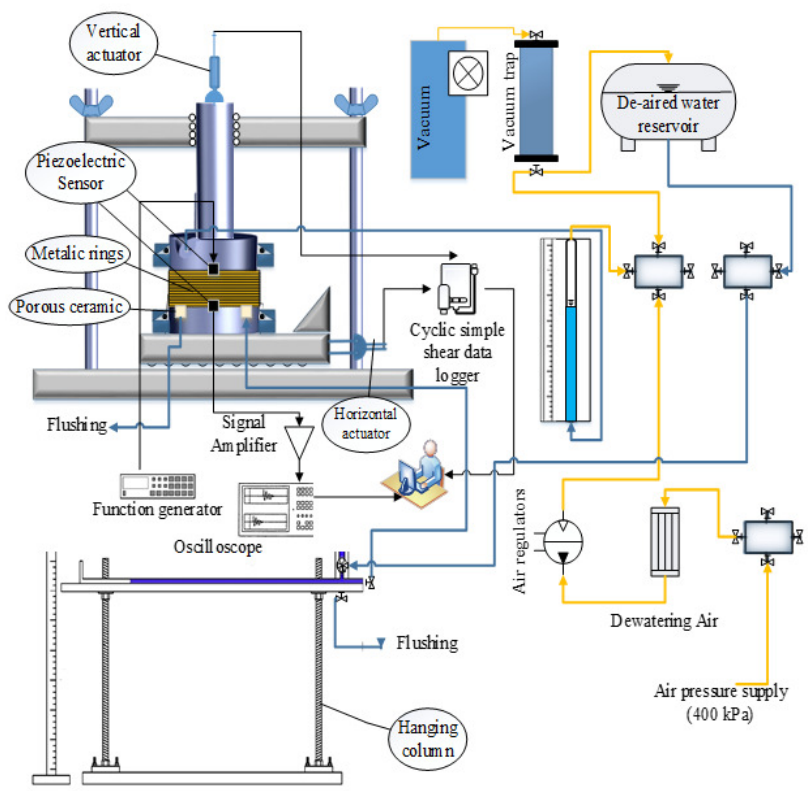

Fig. 1. A schematic view of the newly-modified unsaturated cyclic simple shear apparatus.

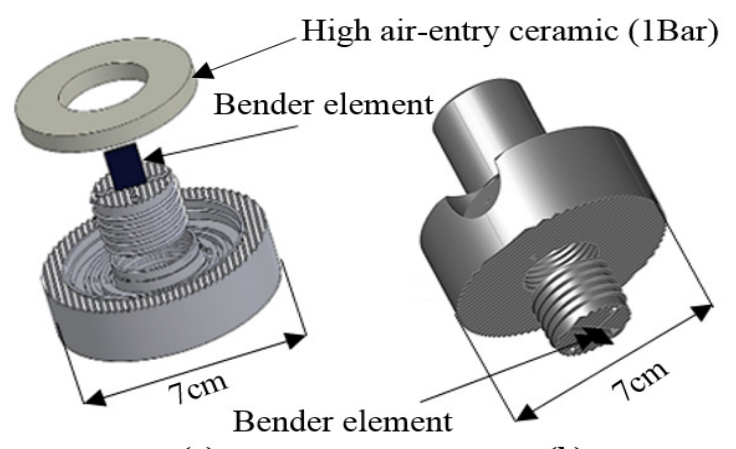

(a)

(b)

Fig. 2. Overview of the (a) base pedestal and (b) top cap after modification for bender element.

\section{Test soil}

The soil used in this study was collected from Babolsar shore in Mazanderan province of the Northern region of Iran. The soil is classified as medium poorly graded sand according to the Unified Soil Classification System (USCS; ASTM D422-63). Some basic index properties of the sand determined by respective ASTM standards are summarised in Table 1. Fig. 3 shows the drying and wetting soil water retention curves (SWRCs) of the sand prepared at $60 \%$ relative density (initial void ratio, $e_{0}$, of 0.71 ) and tested under $50 \mathrm{kPa}$ net vertical stress. The air entry value, residual suction, and residual $S_{r}$ are $2.5 \mathrm{kPa}$, $5 \mathrm{kPa}$ and $7.7 \%$, respectively. The sand has remarkable hydraulic hysteresis after experiencing a cycle of drying and wetting.

Table 1. Some key index properties of the Babolsar sand

\begin{tabular}{|c|c|}
\hline Maximum void ratio $e_{\max }$ & 0.806 \\
\hline Minimum void ratio $e_{\min }$ & 0.575 \\
\hline $\mathrm{D}_{10}$ & 0.15 \\
\hline $\mathrm{D}_{60}$ & 0.28 \\
\hline $\mathrm{D}_{30}$ & 0.2 \\
\hline Coefficient of uniformity, $C_{u}$ & 1.867 \\
\hline Coefficient of curvature, $C_{c}$ & 0.952 \\
\hline Specific gravity $G_{s}$ & 2.793 \\
\hline
\end{tabular}

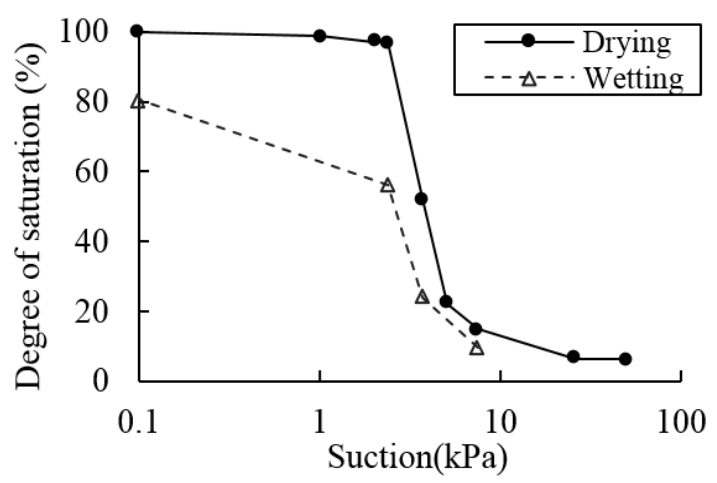

Fig. 3. Measured drying and wetting SWRC of the tested sand

\section{Methods of interpretations}

\subsection{Arrival time of $s$ - and $p$-waves}

To create a p- or s-wave, a transmitter element can be excited to produce a sinusoidal electric pulse through a function generator. An oscilloscope was used to record the signals from both the transmitters and receivers. Since p-wave is the fastest propagation waves compared to s-wave, it would be first detected by the receiver. Hence, its travel time can be determined straightforwardly.

On the contrary, some analysis is needed to determine the travel time of s-wave. As s-wave is propagated in soil, the p-wave would be engendered by the s-wave refraction and the reflection of a rigid boundary. Thus, the p-wave may have already reached before or during the arrival of s-wave [11]. So, the first deflection of the received signal may not correspond to the arrival of the s-wave. Some errors such as the near- 
field, reflection and refraction at the sample boundaries, electromagnetic coupling — crosstalk during test could be prominent, especially for short soil specimen subjected to constraints. Sample size, sensor's characteristic, stiffness of soil, soil type, and characteristics of the transmitted wave can all affect the received wave [12, 13]. The methods of interpreting the arrival time of waves are shown in Fig. 4. The combination of peak-topeak and cross-correlation methods are adopted. In the literature, the time shift corresponding to the maximum cross-correlation is considered as the travel time of the swave [14]. In this research, the value calculated by the cross-correlation method was compared with the time obtained by the peak-to-peak method. If the two values do not differ significantly, the travel time corresponding to the maximum peak of cross-correlation method is considered as the travel time because in this case, the first s-wave received would not be distort by the aforementioned errors. The correct peak obtained from the cross-correlation method does not necessarily relate to the greatest correlation, but one of the peaks can be associated with the first arrival [13]. If the values calculated by the cross-correlation and peak-to-peak methods, however, differ significantly, the time corresponding to the cross-correlation value nearest to the peak-to-peak value is considered as the travel time. In order to investigate the effect of frequency, the s-wave velocity was determined at a range of 14 different frequencies. The value of s-wave velocity that appeared most in these frequency range was taken for further interpretation.

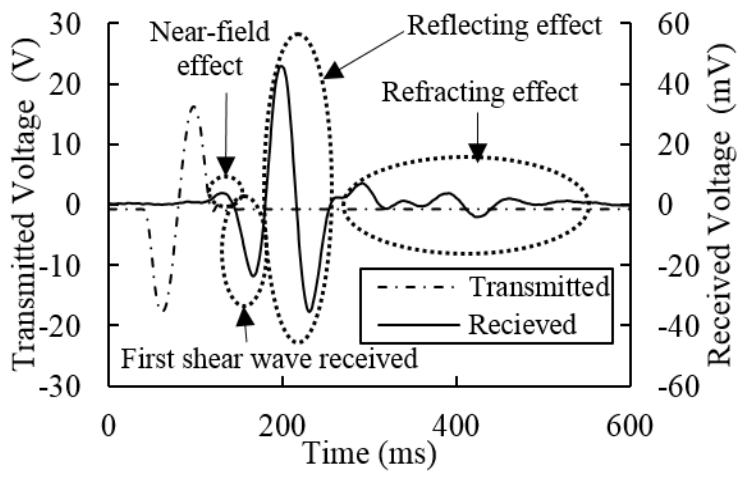

Fig. 4. Interpretation of bender element wave

\subsection{Secant shear modulus}

A typical shear stress $(\tau)$-shear strain $(\gamma)$ curve of a soil after subjecting to a loading-unloading cycle is shown in Fig. 5. In this study, the secant shear modulus at large strain $(G)$ is defined and determined by Eq. 3:

$$
G=\frac{\tau}{\gamma}=\frac{\tau_{\gamma_{\max }}-\tau_{\gamma_{\min }}}{\gamma_{\max }-\gamma_{\min }}
$$

where $\gamma_{\max }$ is the maximum shear strain; $\gamma_{\min }$ is the minimum shear strain; $\tau_{\gamma_{\max }}$ is the shear stress at which the shear strain is maximum; and $\tau_{\gamma_{\min }}$ is the shear stress at which the shear strain is minimum;

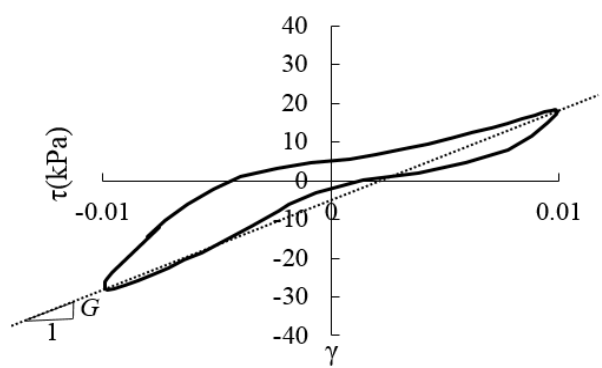

Fig.5. A typical shear stress-shear strain curve of a soil illustrating the definition of secant shear modulus at large strain in this study

\subsection{Material damping}

Quantifying wave attenuation in soils can be complicated because it can be affected by many parameters such as the geometrical spreading, reflections, refractions, backscattering, mode conversion at interfaces and the effects of peripheral electronics [15]. In this study, soil damping was determined by the free vibration decay method [15] which can be expressed as:

$$
D=\frac{\delta}{2 \pi}=\frac{\delta}{\sqrt{4 \pi^{2}+\delta^{2}}}
$$

where $\delta$ is the logarithmic decrement, and the way to obtain it is illustrated in Fig. 6. Note $Z_{n}$ and $Z_{n+1}$ are the amplitude of wave at $n$ and $n+1$ peak respectively.

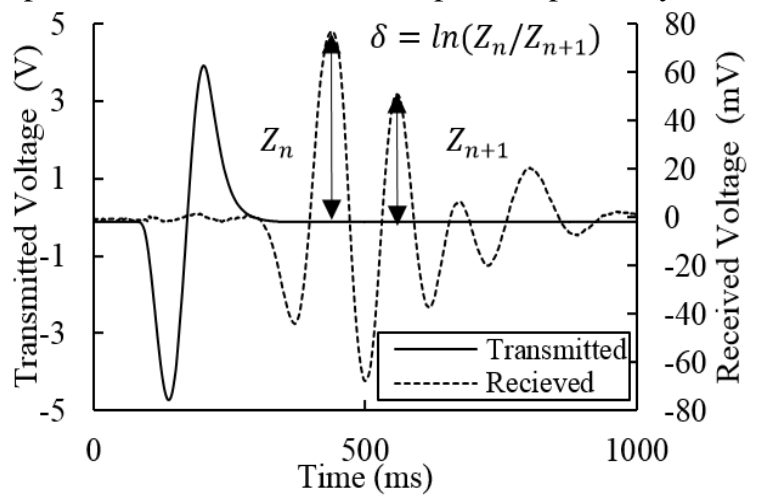

Fig. 6. An example of free vibration of p-wave (frequency $=40$ $\mathrm{kHz}$, wetting path, suction of $5.1 \mathrm{kPa}$ ) under damping.

\section{Test program}

A series of two very-small-strain and six large-strain tests were conducted to study the effects of $S_{r}$ and matric suction on the dynamic properties of the sand (Table 2). For the two very-small-strain tests, an initially saturated sand vertically loaded at a constant value of $50 \mathrm{kPa}$ was subjected to step increases in suction. At each suction equilibrium, a p- or s-wave was transmitted to the unsaturated sand for measuring the $G_{\max }$ and $M$. Effects of suction on $M$ along the wetting path were also studied. As mentioned in section 4.1, for accurately determining s-wave velocity, 14 different frequencies were applied.

For the six large-strain tests, the initially saturated sand subjected to the same constant vertical loading of 
$50 \mathrm{kPa}$ was dried to six different controlled levels of suction. At each suction level, a s-wave was transmitted to assess $G_{\max }$, subsequently followed by a straincontrolled cyclic shear test at a frequency of $1 \mathrm{~Hz}$.

Table 2. A summary of test program.

\begin{tabular}{|c|c|c|c|c|}
\hline Type of test & Large strain & \multicolumn{3}{|c|}{ Very small strain } \\
\hline $\begin{array}{c}\text { Sample } \\
\text { preparation } \\
\text { method }\end{array}$ & \multicolumn{4}{|c|}{ Moist -tamping } \\
\hline Relative density & \multicolumn{4}{|c|}{$60 \%$} \\
\hline Net vertical stress & \multicolumn{4}{|c|}{$50 \mathrm{kPa}$} \\
\hline $\begin{array}{l}\text { Loading } \\
\text { condition }\end{array}$ & $\begin{array}{c}\text { Strain - } \\
\text { controlled } \\
\text { cyclic shear }\end{array}$ & S-wave & \multicolumn{2}{|c|}{ P-wave } \\
\hline $\begin{array}{l}\text { Amplitude of } \\
\text { shear strain }\end{array}$ & $1 \%$ & $<0.0001 \%$ & \multicolumn{2}{|c|}{$<0.0001 \%$} \\
\hline Frequency & $1 \mathrm{~Hz}$ & $\begin{array}{l}3,5,8,10, \\
12,15,18, \\
20,25,30, \\
35,40,50, \\
80(\mathrm{kHz})\end{array}$ & \multicolumn{2}{|c|}{$40 \mathrm{kHz}$} \\
\hline Hydraulic path & Drying & Drying & Drying & Wet \\
\hline
\end{tabular}

\section{Results and discussion}

\subsection{Responses at very-small-strain}

The measured effects of suction and $S_{r}$ on $G_{\max }$ using the interpretation methods adopted in the preceding section are shown in Fig. 7. It can be seen that the $G_{\max }$ did not show any appreciable change until the suction approached the air-entry value of $2.5 \mathrm{kPa}$. Beyond this, a significant increase in $G_{\max }$ was observed as the sand dried further. When attempting to relate $G_{\max }$ with $S_{r}$, after the air entry the shear modulus initiated to increase continuously. In the literature, similar observation was reported. The observed increase in $G_{\max }$ may be attributed to the plastic hardening as soil dries and develops suction $[1,16]$.

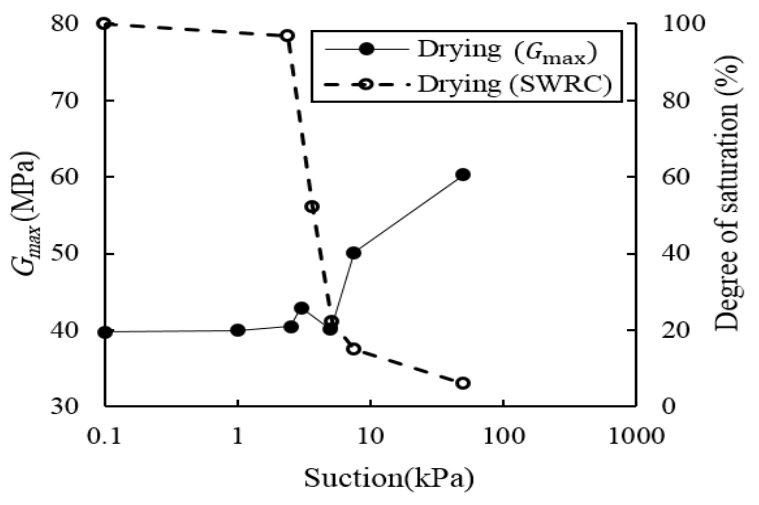

Fig. 7. Effects of suction and $S_{r}$ on $G_{\max }$.

Fig. 8 shows the variations of $M$ with suction along both the drying and wetting paths. As the sand was dried, there was a slight increase in $M$ (though no more than $1 \%$ ) due to the increase of effective stress by suction when the soil remained fully saturated (i.e., before the air-entry value). As the suction increased further to the 'transition zone' of the SWRC, an abrupt drop of $M$ of more than $24 \%$ is found because of the increase in air content in the soil and hence the soil compressibility. Interestingly, $M$ then increased remarkably as the sand was dried beyond the residual suction. This seems to agree with the Biot-Gassmann theory, which predicts that for $S_{r}$ lower than $0.985, V_{p}$ (or $M$ ) in an unsaturated medium would be lower than the corresponding dry medium [17]. Along the wetting path as the sand was wetted in steps, $M$ responded differently. The $M$ first dropped as suction reduced from 50 to $7.5 \mathrm{kPa}$, and it then remained apparently unchanged afterwards. It is postulated that the observed difference of $M$ is because of the differences of distribution of bulk and meniscus water in soil during the drying and wetting processes [18].

The measured variations of the damping ratio with suction is depicted in Fig. 9. It appears that the damping ratio increased abruptly as suction exceeded the air-entry value, but it then remained apparently constant when the soil was dried beyond the residual value. Along the wetting path, the damping ratio, interestingly, first increased as suction reduced to approach to $3.7 \mathrm{kPa}$ but then reduced when the soil was wetted more. Clearly the damping of the sand was hysteretic. A reason to explain the greater soil damping ratio along the wetting path is because of the lower $G_{\max }$ and $M$, hence greater attenuation. In general, wave attenuation in soil can be categorised into four mechanisms: (1) viscous losses and Biot relaxation; (2) squeeze flow between grain contacts, between neighboring pores, and between compressionextension regions; (3) flow due to patchy saturation; and (4) gas bubble vibration [19]. [19] showed that damping ratio increases with an increase in relative humidity (i.e., equivalent to the reduction of soil suction). On the other hand, [20] finds that the p-wave attenuation increases at low levels of $S_{r}$ and it then attains the maximum value between 60 and $90 \%$ of $S_{r}$. As $S_{r}$ continues to increase, p-wave attenuation decreases until reaching the full saturation. In the present findings depicted in Fig. 9, the maximum damping occurred at $3.7 \mathrm{kPa}$ matric suction and $24 \%$ of $S_{r}$. Although the values of suction and $S_{r}$ where the maximum damping is found in this study are different from existing literature, this is somehow expected because of the different soil types investigated among different studies. Nonetheless, the observed trend in the present study is consistent with these studies.

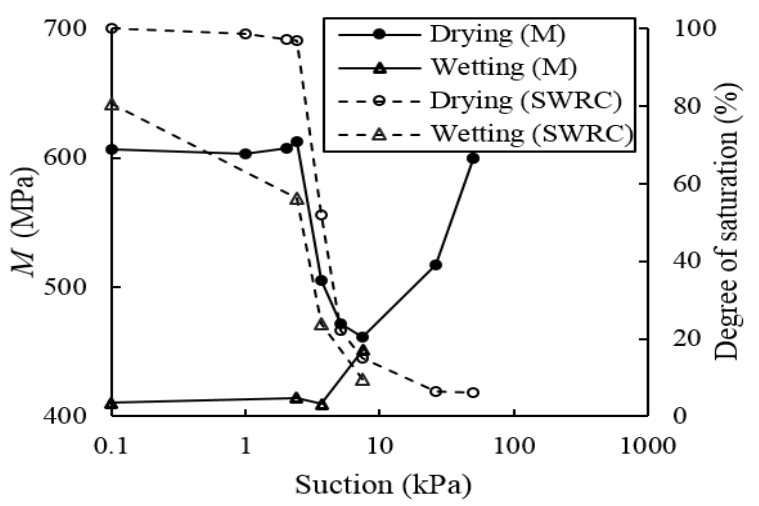

Fig. 8. Effects of suction and $S_{r}$ on the $M$. 


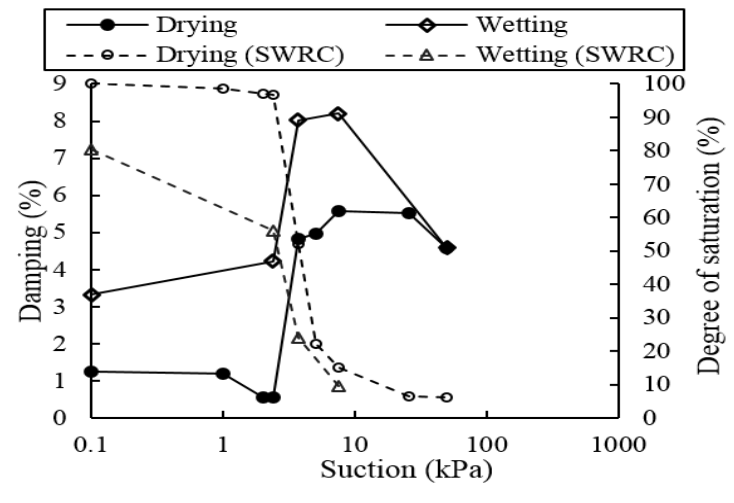

Fig. 9. Effects of suction and $S_{r}$ on damping.

\subsection{Responses at large strain}

The variations of the large-strain $G$ (as determined by Eq. (3)) with the number of cycles at different suctions are depicted in Fig. 10. It can be seen that $G$ stabilized at appropriately at the $150^{\text {th }}$ cycle. Correspondingly, the normalised of $G_{150} / G_{\max }$ (where $G_{150}$ is the large-strain $G$ at the $150^{\text {th }}$ cycle) with suction is given in Fig. 11 . It can be seen that the $G_{150} / G_{\max }$ increases with an increase in suction, yet when reaching the residual zone of the SWRC, the normalised value decreases approximately exponentially until reaching $50 \mathrm{kPa}$ of suction. It seems that the reduction of $G_{150} / G_{\max }$ after $3.7 \mathrm{kPa}$ of suction may be because at residual zone, the water phase in the soil became discontinuous and this may hence lead to a reduction of the contribution of capillary suction [2123].

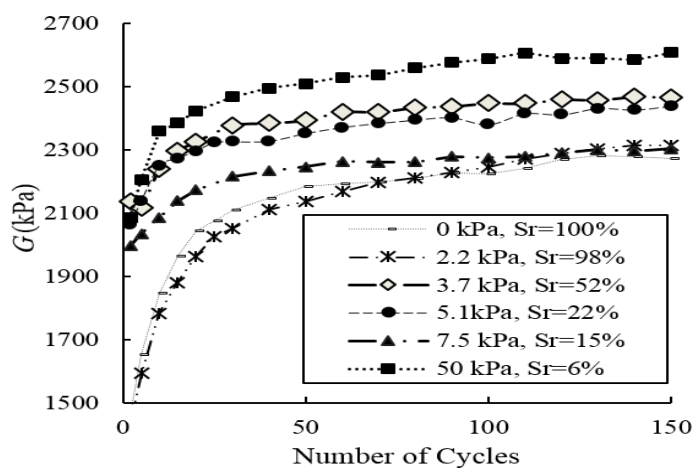

Fig. 10. Mobilisation of large-strain shear modulus with the number of cycles at various suction

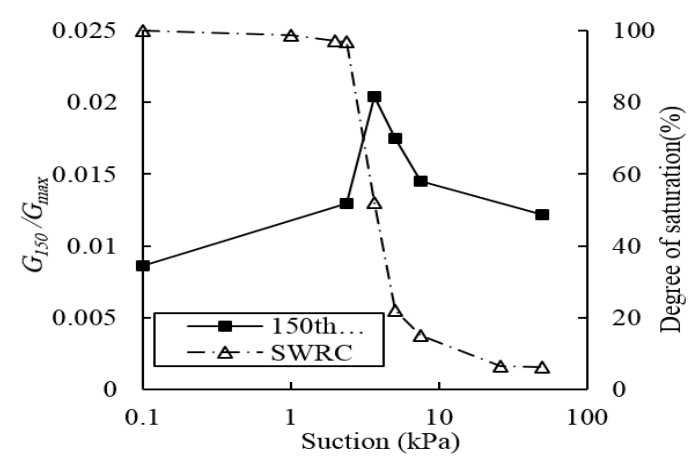

Fig. 11. Effects of suction and $S_{r}$ on mobilised shear modulus at large strain.

\section{Conclusions}

A simple shear apparatus was newly-modified so that both pressure and shear waves can be transmitted and received within an unsaturated soil specimen by mounting a pair of bender elements or ultrasonic sensors on both the top and base pedestal of the apparatus. By using this apparatus, effects of suction and degree of saturation on very-small-strain $(0.0001 \%)$ and largestrain $(1 \%)$ shear modulus and constrain modulus of a sand were quantified and studied. Based on the test results, the following conclusions may be drawn:

(i) The very-small-strain shear moduli showed significant increase as the suction exceeded the airentry value of the sand. Yet, the large-strain shear modulus reduced as the sand was dried further within the 'transition zone', possibly due to any reduction of the effects of capillary suction. Beyond the residual suction, the shear modulus continually increased.

(ii) The very-small-strain constraint modulus, on the other hand, showed significant reduction as the sand became unsaturated and as its suction exceeded the air-entry value. Interestingly, as suction increased further to the transition and residual zones, the effects of suction on the constraint modulus were similar to those on the shear modulus. Along the wetting path, the constraint modulus reduced and it became apparently constant as suction reduced to lower than the air-entry value.

(iii) The damping of sand along the wetting path was always higher than that along the drying path, because the sand was less stiffer along the wetting path.

\section{Acknowledgements}

The authors would like to acknowledge the financial support provided by the Research Grant of Sharif University of Technology as well as the General Research Fund (GRF) \#16212818 funded by the Hong Kong Research Grant Council. The first author is also grateful to Mr. Saeid Hosienpour for his assistance in conducting some of the experiments reported in the present study.

\section{References}

1. T.P. Ngoc, B. Fatahi, and H. Khabbaz, International Journal of Geomechanics 19, 1-21, (2019).

2. A. Mohammadinia, M.M. Disfani, G.A. Narsilio, and L. Aye, Construction and Building Materials 168, 794-804, (2018).

3. S.L.S. Babu and A. Srivastava, Journal of Pipeline Systems Engineering and Practice 1, 33-41, (2010).

4. J.H. Atkinson and G. Sallfors, Proc. of X ECSMFE, Firenze. (1991).

5. F. Jafarzadeh and H. Sadeghi, Soil Dynamics and Earthquake Engineering 32, 26-41, (2012).

6. C. Lee, J.S. Lee, W. Lee, and T.H. Cho, Geotechnical Testing Journal 31, 149-156, (2008).

7. J.U. Youn, Y.W. Choo, and D.S. Kim, Canadian Geotechnical Journal 45, 1426-1438, (2008).

8. E.C. Leong and Z.Y. Cheng, International Journal of 
Geomechanics 16, D4016013, (2016).

9. A. Ahmadinezhad, F. Jafarzadeh, and H. Sadeghi, Soil Dynamics and Earthquake Engineering. 126, 105818, (2019).

10. A.A. Karimzadeh, F. Jafarzadeh, and A. Ahmadinezhad, Advance Researches in Civil Engineering. 1, 8-15, (2019).

11. J.-S. Lee and J.C. Santamarina, Journal of Geotechnical and Geoenvironmental Engineering. 131, 1063-1070, (2005).

12. J. Arroyo, M., Muir Wood, D., Greening, P. D., Medina, L. \& Rio, Géotechnique. 39-52, (2006).

13. A.K.M. Mohsin and D.W. Airey, Proc. Prefailure Deformation Characteristics of Geomaterials, Lyon, France. 73-80, (2003).

14. G. Viggiani and J.H. Atkinson, Géotechnique 47, 873-877, (1997).

15. K.H. Stokoe and J.C. Santamarina, In: ISRM International Symposium. International Society for Rock Mechanics (2000).

16. A. Khosravi, S. Salam, J.S. McCartney, and A. Dadashi, International Journal of Geomechanics. 16, 1-10, (2016).

17. M. Emerson and P. Foray, Acta Geotechnica. 1, 167-177, (2006).

18. L.A. George, M.M. Dewoolkarb, and D. Znidarcicc, Vadose Zone Journal. 8, 633-642, (2009).

19. Y.H. Wang and J.C. Santamarina, Granular Matter. 9, 365-376, (2007).

20. K.W. Winkler and A. Nur, Geophysics. 47, 1-15, (1982).

21. X. Qian, D.H. Gray, and R.D. Woods, Journal of Geotechnical and Geoenvironmental Engineering. 119, 295-314, (1993).

22. K.N. Le and M. Ghayoomi, Geotechnical Testing Journal. 40, 381-395, (2017).

23. F. Jafarzadeh, A. Ahmadinezhad, and H. Sadeghi, Journal of Scientia Iranica 98, (2019). 